\title{
Remoção de matéria orgânica do esgoto em solo de wetland construído
}

\author{
Removal of organic matter fraction from sewage in constructed wetland soil
}

Selma Cristina da Silva', Ricardo Silveira Bernardes², Maria Lucrécia Gerosa Ramos ${ }^{3}$

口-

\section{RESUMO}

Nos sistemas wetlands construídos os meios suportes tradicionalmente utilizados são areia grossa, cascalho, pedregulho e brita. Nesta pesquisa, foi utilizado o solo natural (Latossolo Vermelho-Amarelo) misturado com areia média, plantado (wetlands construídos) com arroz irrigado (Oryza sativa L.) ou não (controles). O objetivo foi avaliar a remoção de DBO dos esgotos domésticos pelos processos de imobilização do carbono da biomassa microbiana e da respiração basal (atividade microbiana). Foram utilizados três sistemas operando com taxas de aplicação hidráulica de 4, 8 e 15 cm.d! Cada sistema apresentava três unidades wetlands construídos e três controles, as quais eram alimentadas com esgotos para tratamento, por fluxo vertical em regime não saturado, nas segundas, quartas e sextas-feiras, ficando os outros dias em descanso para aeração do solo. Os resultados mostraram que os solos foram capazes de reduzir as concentrações de DBO afluentes significativamente, com eficiência de remoção variando entre 97 e 99\%. A remoção se deu pela mineralização da matéria orgânica e seu acúmulo no solo. Durante o início da aplicação do esgoto, houve inicialmente uma redução nos teores de matéria orgânica nativa do solo que passaram a aumentar paulatinamente com a aplicação dos esgotos. A atividade dos microrganismos decompositores aumentou proporcionalmente à taxa de aplicação de esgoto, porém taxas elevadas indicaram uma tendência à inibição dessa atividade. Nos solos das unidades controles, houve menores perdas de $\mathrm{CO}_{2}$ para a atmosfera, indicando uma maior imobilização do carbono nos solos.

Palavras-chave: esgoto doméstico; wetlands construídos; remoção de matéria orgânica; solo; atividade microbiana.

\begin{abstract}
On constructed wetlands systems, the support media usually used are coarse sand, gravel, crushed stone and gravel. In this research, we used the natural soil (Oxisol) mixed with medium sand, planted with rice (Oryza sativa L.) and a control without plants. The objective was to evaluate the BOD removal through the immobilization of microbial biomass, basal respiration (microbial activity) and total organic carbon of the soil. We used three systems, operating with hydraulic application rates of 4,8 e $15 \mathrm{~cm} \cdot \mathrm{d}^{-1}$. Each system had three units constructed wetlands and three controls, which were fed with sewage, with vertical flow unsaturated conditions, on Mondays, Wednesdays and Fridays, leaving the system with no feed on the rest of the days, for soil aeration. The results showed the soils were able to reduce significantly the BOD concentration, with removal efficiency ranging between 97 and 99\%. The removal was due to the mineralization of organic matter and its accumulation in the soil. During the system start up operation, there was reduction in native soil organic matter, which started to increase gradually by the sewage application. The activity of microbial decomposers increased proportionally to the rate of sewage application, but high rates showed a trend to inhibition of this activity. In soils units controls showed minor loss of $\mathrm{CO}_{2}$ to the atmosphere, indicating a higher carbon immobilization in soils.
\end{abstract}

Keywords: sewage; constructed wetlands; organic substance removal; soil; microbial activity.

\section{INTRODUÇÃO}

Os wetlands construídos são sistemas que utilizam o princípio de solo úmido cultivado onde o complexo solo-micorganismos-rizosfera da planta é responsável pela despoluição das águas residuárias. Esses sistemas podem desempenhar funções semelhantes ao tratamento convencional dos esgotos domésticos por meio de processos físicos, químicos e biológicos. Porém, o tratamento com sistemas wetlands construídos é

mais indicado para pequenas comunidades, inclusive as comunidades rurais. Nesse tipo de sistema é possível obter tratamento terciário em uma única unidade, com custos bastante reduzidos, e também utilizar plantas produtoras de grãos, agregando benefícios econômicos, financeiros e ambientais.

Os benefícios econômicos e financeiros tanto podem ser obtidos com a comercialização da biomassa vegetal e/ou dos grãos produzidos

'Doutora em Tecnologia Ambiental e Recursos Hídricos pela Universidade de Brasília (UnB). Professora Adjunto III do Centro de Ciências Exatas e Tecnológicas da Universidade Federal do Recôncavo da Bahia (CETEC/UFRB) - Cruz das Almas (BA), Brasil.

${ }^{2}$ Doutor em Agricultura e Ciências Ambientais pela Wageningen Agricultural University (WAU). Professor do Departamento de Engenharia Civil da UnB - Brasília (DF), Brasil. ${ }^{3} \mathrm{PhD}$ em Ecofisiologia Vegetal pela University of Dundee. Professora da Faculdade de Agronomia e Medicina Veterinária da UnB - Brasília (DF), Brasil.

Endereço para correspondência: Selma Cristina da Silva - Universidade Federal do Recôncavo da Bahia (UFRB), Campus Universitário de Cruz das Almas - Centro -

44380-000 - Cruz das Almas (BA), Brasil - E-mail: scsilvaOO@yahoo.com.br.

Recebido: 23/11/2011- Aceito: 17/09/2015 - Reg. ABES: 75357 
quanto com a redução dos custos pela utilização de um meio suporte encontrado no próprio local, sem que haja a necessidade de compra e transporte desse material. Os recursos arrecadados podem ser empregados na administração e manutenção dos sistemas. Como benefícios ambientais, têm-se a não contaminação dos corpos d'água superficiais pelo lançamento de esgotos com concentrações elevadas de poluentes e pelo carreamento, por meio do escoamento superficial, de substâncias presentes no solo em razão da aplicação de fertilizantes químicos em áreas agrícolas e também pela redução da retirada de água dos rios para a irrigação de culturas. Essas características conferem aos sistemas wetlands construídos uma autossustentabilidade.

Os três tipos fundamentais de sistemas wetlands construídos baseados em macrófitas aquáticas, segundo Vymazal (1998), são: flutuantes, submersas e emergentes.

Os sistemas de plantas emergentes são os mais utilizados e podem ser classificados segundo o fluxo como superficiais e subsuperficial horizontal, vertical e híbrido, ou misto (combinação de fluxo vertical com fluxo horizontal). Os sistemas de fluxo superficial são mais comumente encontrados nos Estados Unidos do que na Europa, embora venha sendo praticado na Holanda desde a década de 1970.

Geralmente, os meios suportes utilizados nos wetland construídos de fluxo subsuperficial são: areia grossa, cascalho, pedregulho e brita. Porém, já foram avaliados outros materiais inertes como pneus e conchas de ostra, os quais apresentaram eficiências satisfatórias na remoção de poluentes.

Essa tecnologia apresenta condições para formar um sistema de tratamento bastante completo e eficiente. O sucesso já foi comprovado pelo seu histórico, pois os primeiros estudos sobre wetlands construídos iniciaram-se na Europa na década de 1950 e nos Estados Unidos na década de 1960 (EPA, 2006), aumentando as pesquisas entre 1970 e 1980. No Brasil, a utilização dos wetlands construídos vem sendo difundida lentamente e estudada por diversas instituições de pesquisa. Os sistemas wetlands construídos que utilizam materiais tradicionais como meio suporte apresentam eficiências satisfatórias na remoção de Demanda Bioquímica de Oxigênio (DBO) de 77,8 e 83,3\% (MEIRA, 2004) e de 80\% (CALIJURI et al., 2009). Com o uso de plásticos, Sklarz et al. (2009) obtiveram eficiência média de 95\%, e, com camadas de britas de diferentes granulometrias e areia, Morari e Giardini (2009) obtiveram eficiência maior que 86\%. Trang et al. (2010) encontraram eficiências de remoção de DBO em wetlands construídos de fluxo horizontal com meio suporte de areia variando entre 65 e $83 \%$.

No entanto, as avaliações do desempenho de remoção de DBO das unidades wetlands construídos têm sido comumente observadas somente por meio de análises de amostras do seu afluente e dos efluentes. Nesse trabalho, buscou-se avaliar a remoção da matéria orgânica (MO) nesses sistemas com meio suporte de solo local (Latossolo
Vermelho-Amarelo) totalmente distrófico, visando observar o seu desempenho no tratamento e a possibilidade do alcance da sua fertilização.

A MO é a principal responsável pela Capacidade de Troca Catiônica (CTC) dos solos. Ela atua como reservatório de nutrientes (ex.: cálcio, magnésio e potássio) e aumenta a absorção destes pelas plantas, minimizando suas perdas para as camadas mais profundas do solo. Os benefícios promovidos ao solo pela adição de $\mathrm{MO}$ podem ser mineralização de nutrientes para a absorção pelas plantas; melhoria da capacidade tampão do solo pelo aumento da CTC; melhoria na estrutura do solo (estado de agregação das partículas); maior capacidade de absorção e armazenamento de água e melhoria na aeração. Contribui também para: o incremento da atividade biológica, favorecendo o desenvolvimento do sistema radicular, promovendo maior produtividade às culturas (SILVA, 2005); o fornecimento do carbono como fonte de energia para os microrganismos e a promoção da ciclagem de nutrientes; a interação com metais, óxidos e hidróxidos metálicos, atuando como trocador de íons e estocadores de nitrogênio, fósforo e enxofre (MARTINAZZO, 2006).

Nos esgotos domésticos, a MO é encontrada em solução - representada pelos sólidos orgânicos dissolvidos (rapidamente biodegradáveis) - e em suspensão - relativa aos sólidos suspensos no meio líquido (lentamente biodegradáveis). Nos sistemas wetlands construídos, os principais responsáveis pela remoção da matéria orgânica são os microrganismos (aeróbios, facultativos e anaeróbios) que a utilizam, retirando dela a energia necessária para síntese celular e transformando os compostos orgânicos, mineralizando nutrientes ou formando as substâncias húmicas do solo (MOREIRA \& SIQUEIRA, 2002).

Nesse trabalho, procurou-se estudar o desempenho dos sistemas wetlands construídos de fluxo vertical com meio suporte de solo (Latossolo Vermelho-Amarelo), plantados com arroz irrigado (Oryza sativa L.) e controles (sem planta), operados com diferentes taxas de aplicação hidráulica de esgotos na remoção de DBO, observando os efeitos dessa remoção por meio da imobilização do $\mathrm{C}$ da biomassa microbiana, da respiração basal (atividade microbiana) e do carbono orgânico total do solo.

\section{METODOLOGIA}

A pesquisa foi desenvolvida a partir de 3 sistemas experimentais, cada um deles com 6 unidades - 3 plantadas (wetlands construídos) e 3 sem plantas (controles), totalizando 18 (dezoito) unidades experimentais.

Cada unidade experimental era representada por uma bombona plástica cilíndrica (tambor) com capacidade de $200 \mathrm{~L}$, altura de $0,87 \mathrm{~m}$, diâmetro interno de 0,60 m e com área superficial de 0,26 $\mathrm{m}^{2}$. A escolha desse tipo de material foi em função do seu custo e da sua capacidade de resistência ao peso do meio suporte saturado. Os sistemas 1, 2 e 3 foram operados sob condição não saturada com taxa de aplicação 
hidráulica variável, respectivamente, de 4,8 e $15 \mathrm{~cm} \cdot \mathrm{d}^{-1}$, e com alimentação intermitente alternada. Essas taxas foram aplicadas sobre a superfície dos leitos filtrantes nas segundas, quartas e sextas-feiras, manualmente com um regador, durante os 10 meses de operação dos sistemas. Os dias de descanso favoreciam a aeração dos solos, necessária à oxidação do nitrogênio total a nitrato (nitrificação).

Os sistemas foram instalados no pátio da Estação de Tratamento de Esgoto de Brasília (ETEB) Norte, da Companhia de Saneamento Ambiental do Distrito Federal (CAESB), próximo ao Decantador Primário (DP), unidade da qual era captado o esgoto a ser tratado. Houve a necessidade de um tratamento prévio para remoção de sólidos em suspensão, uma vez que o meio suporte era de solo, a fim de evitar sua rápida colmatação do solo.

O meio suporte utilizado foi o solo natural da cidade de Brasília (Latossolo Vermelho-Amarelo) misturado com areia média, na proporção, respectivamente, de 1,0:1,5. Essa mistura garantiu uma condutividade hidráulica $(\mathrm{K})$ adequada para manter a vazão a ser tratada. Foram colocados, em média, $290 \mathrm{~kg}$ de solo em cada unidade, com altura igual a $0,55 \mathrm{~m}$.

A cultura utilizada nos wetlands construídos foi o arroz (Oryza sativa L.), semeado na Estação Biológica da Universidade de Brasília (UnB) em um canteiro de $5 \mathrm{~m}$ de comprimento, $0,80 \mathrm{~m}$ de largura e 0,10 $\mathrm{m}$ de altura, deixando-se $0,01 \mathrm{~m}$ de borda livre para evitar perdas de solo e de água durante a irrigação. O solo foi adubado com N:P:K (4:14:8 g.kg de solo $^{-1}$ ) e posteriormente foram abertas covas no solo, a uma profundidade de aproximadamente $1,0 \mathrm{~cm}$, ao longo do canteiro e cada uma recebeu 7 sementes. $\mathrm{O}$ canteiro foi irrigado 2 vezes por dia, e a germinação ocorreu aos 9 dias após a semeadura. $\mathrm{O}$ transplantio das mudas para as unidades experimentais foi efetuado aos 25 dias após a germinação, quando essas unidades passaram a receber o esgoto para tratamento.

A cultura do arroz foi transplantada em dezembro de 2005, e em abril de 2006 (final do primeiro ciclo da cultura) coletou-se a parte aérea da planta, deixando as raízes presas ao solo das unidades experimentais para que rebrotassem. A segunda coleta das plantas com o seu sistema radicular ocorreu em setembro de 2006.

O monitoramento dos processos de remoção de matéria orgânica foi realizado por meio de análises de amostras do esgoto afluente e dos efluentes realizadas com periodicidade quinzenal e, também, de amostras de solo antes e após a aplicação do esgoto para tratamento.

O procedimento de coleta, preservação e análises das amostras dos afluentes e efluentes aos sistemas foi realizado com base nas recomendações e métodos do Standard Methods for Examination of Water and Wastewater (APHA-AWWA-WPCF, 1985).

A coleta das amostras de solo foi feita em cada unidade experimental, nas camadas de $0-5 \mathrm{~cm}$ do solo, após o primeiro ciclo do arroz, e nas camadas de $0-5$ e $5-20 \mathrm{~cm}$, no final do segundo ciclo da cultura. No segundo ciclo da cultura, foi analisada também a camada de 5-20 cm para se avaliar se houve acúmulo significativo de matéria orgânica em toda a camada arável do solo. A metodologia de análise seguiu os procedimentos do "Manual de Métodos de análise química para avaliação da fertilidade do solo" da EMBRAPA.

O carbono da biomassa microbiana foi determinado pelo método de fumigação e extração (VANCE; BROOKES; JENKINSON, 1987), e a respiração basal do solo $\left(\mathrm{CO}_{2}\right.$ perdido para a atmosfera) pelo método de Alef e Nannipierre (1995).

O delineamento experimental foi em blocos ao acaso, com 3 repetições, em esquema fatorial 3 x 2, ou seja, 3 taxas de aplicação hidráulica dos esgotos $\left(4,8\right.$ e $\left.15 \mathrm{~cm} \cdot \mathrm{d}^{-1}\right)$ e 2 sistemas, um com planta (wetlands construídos) e outro sem planta (controles). A comparação de médias foi feita pelo teste de Tukey $(\mathrm{p}<0,05)$.

\section{RESULTADOS E DISCUSSÃO}

A remoção da matéria orgânica (MO) presente no esgoto, possivelmente, ocorreu a partir do processo de mineralização pelos microrganismos decompositores, produzindo e liberando $\mathrm{CO}_{2}$ para a atmosfera, e pelo seu acúmulo do carbono orgânico no solo.

No período estudado, as eficiências médias de remoção das unidades wetlands construídos e controles variaram, aproximadamente, entre 97 e 99\%, não havendo diferenças significativas de remoção entre os sistemas que receberam diferentes taxas de aplicação hidráulica e entre os wetlands construídos e controles. As concentrações de DBO nos efluentes (Tabela 1) foram comparáveis àquelas estabelecidas pela Resolução CONAMA 357/05 (BRASIL, 2005) para rios de classes 1 e 2 que devem conter, no máximo, respectivamente, 3 e 5 mg.L $\mathrm{L}^{-1}$ de DBO. Portanto, muito abaixo dos padrões de lançamento estabelecidos pela União Europeia, Directive 91/271/EEC, pelas Deliberações estaduais brasileiras (NASCIMENTO, 1998) e pela Resolução CONAMA n ${ }^{\circ}$

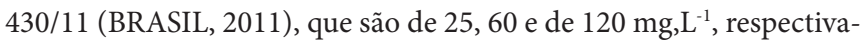
mente, para rios de Classes 1, 2 e 3.

Fia et al. (2010), em wetlands construídos de fluxo horizontal com meio suporte de brita zero plantado com Taboa (Typha sp.) para tratamento de água residuária do processamento dos frutos do cafeeiro, observaram que o aumento nas taxas de aplicação orgânica proporcionou redução da eficiência de remoção de DBO, tendo o sistema que recebeu menor taxa de aplicação orgânica apresentado maior eficiência de remoção de DBO (63\%). Portanto, as características da água residuária a ser tratada influenciam na eficiência de remoção da MO.

As Figuras 1 a 3 mostram o desempenho na remoção de DBO dos três sistemas ao longo do período de sua operação. Nota-se que a variabilidade nas concentrações efluentes foi mais acentuada durante o segundo ciclo da cultura tanto nos wetlands construídos como nos controles.

$\mathrm{O}$ aumento das concentrações de DBO nos efluentes durante o segundo ciclo da cultura pode ter ocorrido devido ao corte da planta 
Tabela 1 - Concentrações médias afluentes e efluentes \pm desvio padrão, e eficiências médias de remoção de DBO das unidades wetlands construídos e controles dos sistemas 1,2 e 3.

\begin{tabular}{|c|c|c|c|c|c|c|c|c|c|}
\hline \multirow{4}{*}{ Sistema } & \multirow{4}{*}{$\underset{\left(\mathrm{cm}_{\mathrm{h}} \mathrm{d}^{-1}\right)}{\mathrm{q}}$} & \multicolumn{8}{|c|}{ Concentrações efluentes de DBO (mg. $\left.\mathrm{L}^{-1}\right)$ e eficiências de remoção (\%) } \\
\hline & & \multicolumn{4}{|c|}{$1{ }^{\circ} \mathrm{Ciclo}(\mathrm{dez} / 05$-maio/06) } & \multicolumn{4}{|c|}{$2^{\circ}$ Ciclo (maio/06-set/06) } \\
\hline & & \multicolumn{2}{|c|}{ Wetland construído } & \multicolumn{2}{|c|}{ Controles } & \multicolumn{2}{|c|}{ Wetland construído } & \multicolumn{2}{|c|}{ Controles } \\
\hline & & $\mathrm{mg} \cdot \mathrm{L}^{-1}$ & $\%$ & $\mathrm{mg} \cdot \mathrm{L}^{-1}$ & $\%$ & $\mathrm{mg} \cdot \mathrm{L}^{-1}$ & $\%$ & $\mathrm{mg} \cdot \mathrm{L}^{-1}$ & $\%$ \\
\hline 1 & 4 & $1,58 \pm 1,41$ & 98,95 & $1,44 \pm 1,64$ & 99,04 & $3,89 \pm 2,78$ & 97,44 & $2,54 \pm 1,95$ & 98,33 \\
\hline 2 & 8 & $1,17 \pm 0,78$ & 99,22 & $1,60 \pm 1,12$ & 98,94 & $3,48 \pm 2,04$ & 97,71 & $1,22 \pm 1,15$ & 99,19 \\
\hline 3 & 15 & $1,32 \pm 1,30$ & 99,12 & $0,96 \pm 0,86$ & 99,36 & $1,75 \pm 1,29$ & 98,85 & $2,15 \pm 1,50$ & 98,56 \\
\hline \multicolumn{2}{|c|}{$\begin{array}{l}\text { Concentração de DBO afluente } \\
\left.\text { (mg.L }{ }^{-1}\right)\end{array}$} & \multicolumn{4}{|c|}{$150,45 \pm 30,30$} & \multicolumn{4}{|c|}{$152,00 \pm 35,21$} \\
\hline
\end{tabular}

$\mathrm{q}_{\mathrm{h}}$ : Taxa de aplicação hidráulica do esgoto; DBO: Demanda Bioquímica de Oxigênio.

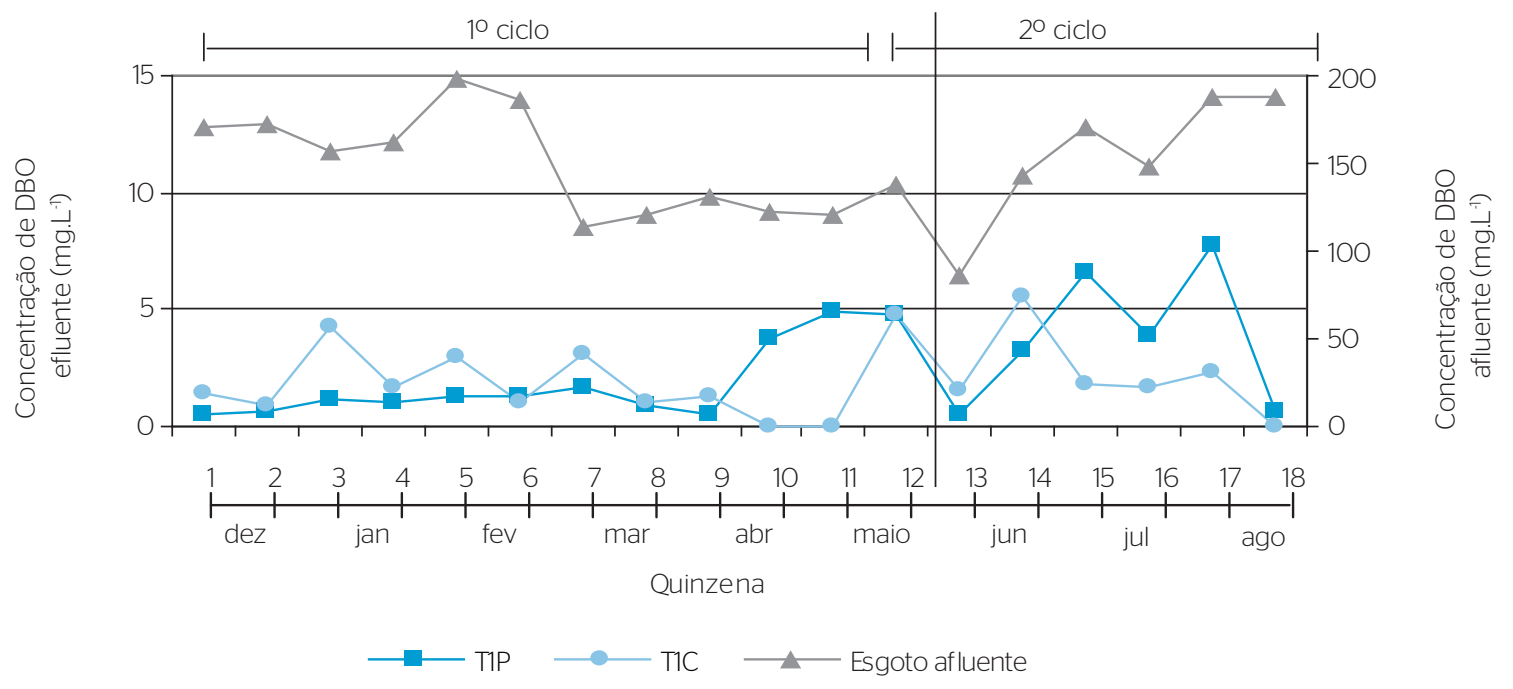

Figura 1 - Concentrações médias de DBO nos afluentes (esgotos) e efluentes das unidades wetlands construídos (T1P) e controles (T1C) do Sistema 1 no período de dezembro de 2005 a agosto de 2006.

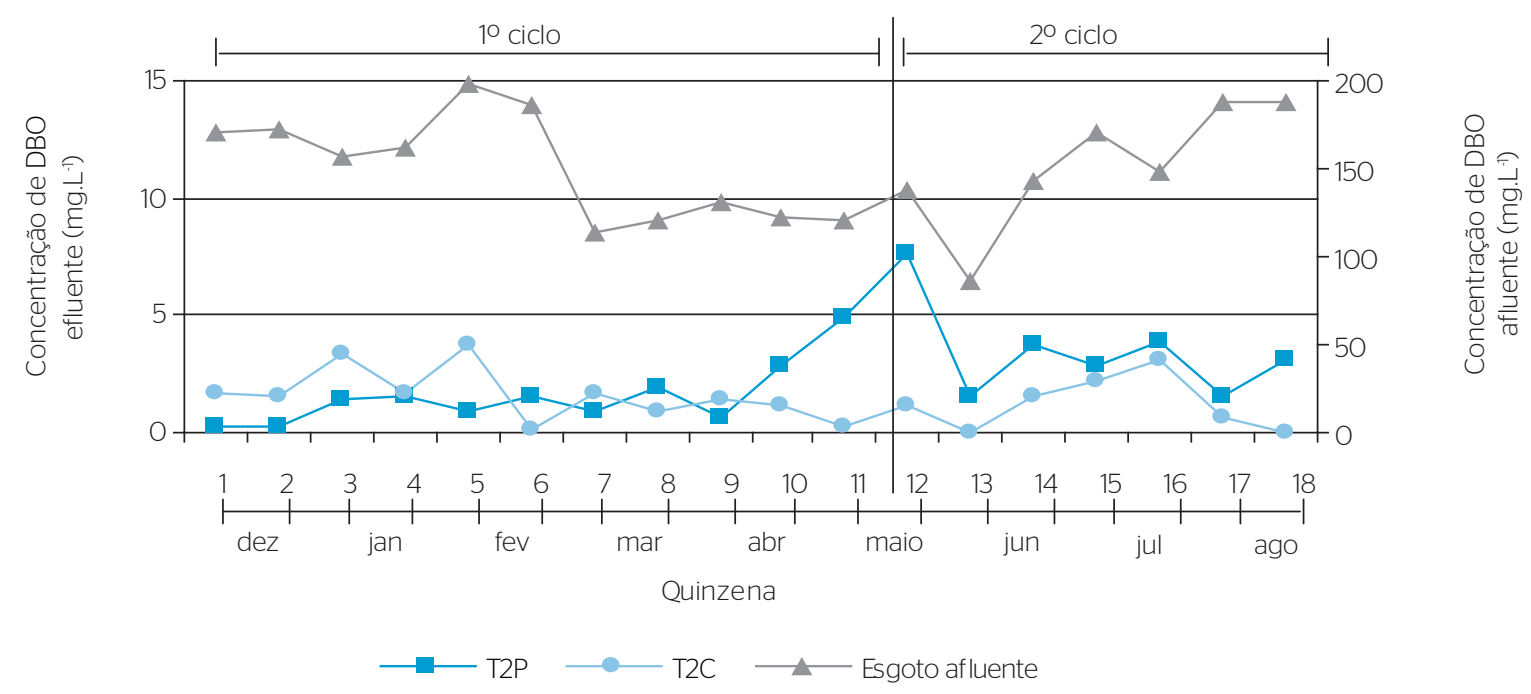

Figura 2 - Concentrações médias de DBO nos afluentes (esgotos) e efluentes das unidades wetlands construídos (T2P) e controles (T2C) do Sistema 2 no período de dezembro de 2005 a agosto de 2006. 
para rebrota, ou ao constante aporte de MO via aplicação do esgoto, que favoreceu a lixiviação de uma fração um pouco maior para os efluentes. Isso poderá ser controlado pelo estabelecimento de formas de manejo que possam manter a matéria orgânica no solo para ser utilizada pelas plantas.

A MO aportada via aplicação do esgoto para tratamento foi mineralizada e acumulada no solo paulatinamente, como pode ser observados nos itens subsequentes.

\section{Acúmulo e mineralização \\ da matéria orgânica dos solos}

Durante os cinco meses iniciais de operação dos sistemas (primeiro ciclo da cultura do arroz), houve redução dos teores de MO na camada de $0-5 \mathrm{~cm}$ dos solos (Tabela 2). Essa redução pode ter ocorrido em razão da adição de resíduos orgânicos via esgoto, que estimulou a atividade dos microrganismos decompositores, os quais, além de utilizarem a matéria orgânica presente no esgoto, também podem ter degradado a matéria orgânica nativa do solo (KUZYAKOV; STAHR; FRIEDEL, 2000). Esse processo é denominado efeito priming positivo (SILVA, 2005), que é caracterizado pela intensa atividade microbiana proveniente do esgoto adicionado ao solo e àquela autóctone do solo (TRUU; JUHANSON; TRUU, 2009).

Nota-se nos resultados da Tabela 2 que a MO remanescente no solo dos wetlands construídos foi significativamente maior do que aquela dos controles somente no sistema que recebeu menor taxa de aplicação de esgoto $\left(4 \mathrm{~cm} \cdot \mathrm{d}^{-1}\right)$. Considerando as taxas de aplicação hidráulica de esgoto para tratamento, o acúmulo da MO no solo foi significativamente maior nos solos dos wetlands construídos e dos controles que receberam maiores taxas de aplicação de esgoto $\left(8\right.$ e 15 cm.d $\left.\mathrm{d}^{-1}\right)$.
Após o segundo ciclo da cultura do arroz, nas camadas de $0-5 \mathrm{~cm}$, houve aumento da matéria orgânica do solo em todos os três sistemas, sejam os plantados com o arroz (wetlands construídos) ou os sem planta (controles), comparados com o solo antes da aplicação do esgoto. Porém, esses valores foram significativamente maiores nos sistemas com a presença da planta do arroz (wetlands construídos), independentemente da taxa de esgoto aplicada (Tabela 3).

$\mathrm{O}$ aumento da MO do solo pode ter ocorrido devido: à constante adição de esgoto e às raízes vivas que liberam compostos de carbono no solo e/ou à lise de células das raízes (MOREIRA \& SIQUEIRA, 2002); à pequena fração dos produtos dessa mineralização (compostos fenólicos solúveis e compostos lignificados parcialmente transformados) que ficou retida; ou aos polissacarídeos (mucilagens) provenientes do metabolismo microbiano de resíduos vegetais e de exsudação

Tabela 2-Quantidade de Matéria Orgânica do Solo adicionada via aplicação do esgoto e aquela remanescente na camada de 0-5 cm do solo após o primeiro ciclo da cultura do arroz (dezembro de 2005 a abril de 2006).

\begin{tabular}{|c|c|c|c|c|c|}
\hline \multirow{2}{*}{ Sistema } & \multirow{2}{*}{$\underset{\left(\mathrm{cm}^{\left.-\mathrm{d}^{-1}\right)}\right.}{\mathrm{q}_{\mathrm{h}}}$} & \multicolumn{2}{|c|}{$\begin{array}{c}\text { Teor de MO } \\
\text { (g MO.kg de solo-1) }\end{array}$} & \multicolumn{2}{|c|}{$\begin{array}{c}\text { MO remanescente no } \\
\text { solo } \\
\text { (g MO.kg de solo-1) }\end{array}$} \\
\hline & & Efluente & $\begin{array}{l}\text { Solo + } \\
\text { esgoto }\end{array}$ & $\begin{array}{l}\text { Wetland } \\
\text { construído }\end{array}$ & Controle \\
\hline 1 & 4 & 0,33 & 7,73 & $5,20 \mathrm{bA} A^{*}$ & $3,27 a A$ \\
\hline 2 & 8 & 0,73 & 8,13 & $6,47 a \mathrm{~B}$ & $6,63 \mathrm{aB}$ \\
\hline 3 & 15 & 1,34 & 8,74 & $7,20 a B$ & $7,07 a B$ \\
\hline
\end{tabular}

$\mathrm{MO}$ no solo antes da aplicação do esgoto ( $\mathrm{g} \cdot \mathrm{kg}^{\mathrm{k}}$ de solo-1) $=7,40$

$\mathrm{q}_{\mathrm{h}}$ : Taxa de aplicação hidráulica do esgoto; MO: Matéria Orgânica; MOS: Matéria Orgânica do Solo.

*Médias seguidas pelas mesmas letras minúsculas nas linhas (comparação entre wetlands e controles) e as maiúsculas nas colunas (comparação entre as taxas de aplicação do efluente) não diferem entre si pelo teste de Tukey $(p<0,05)$.

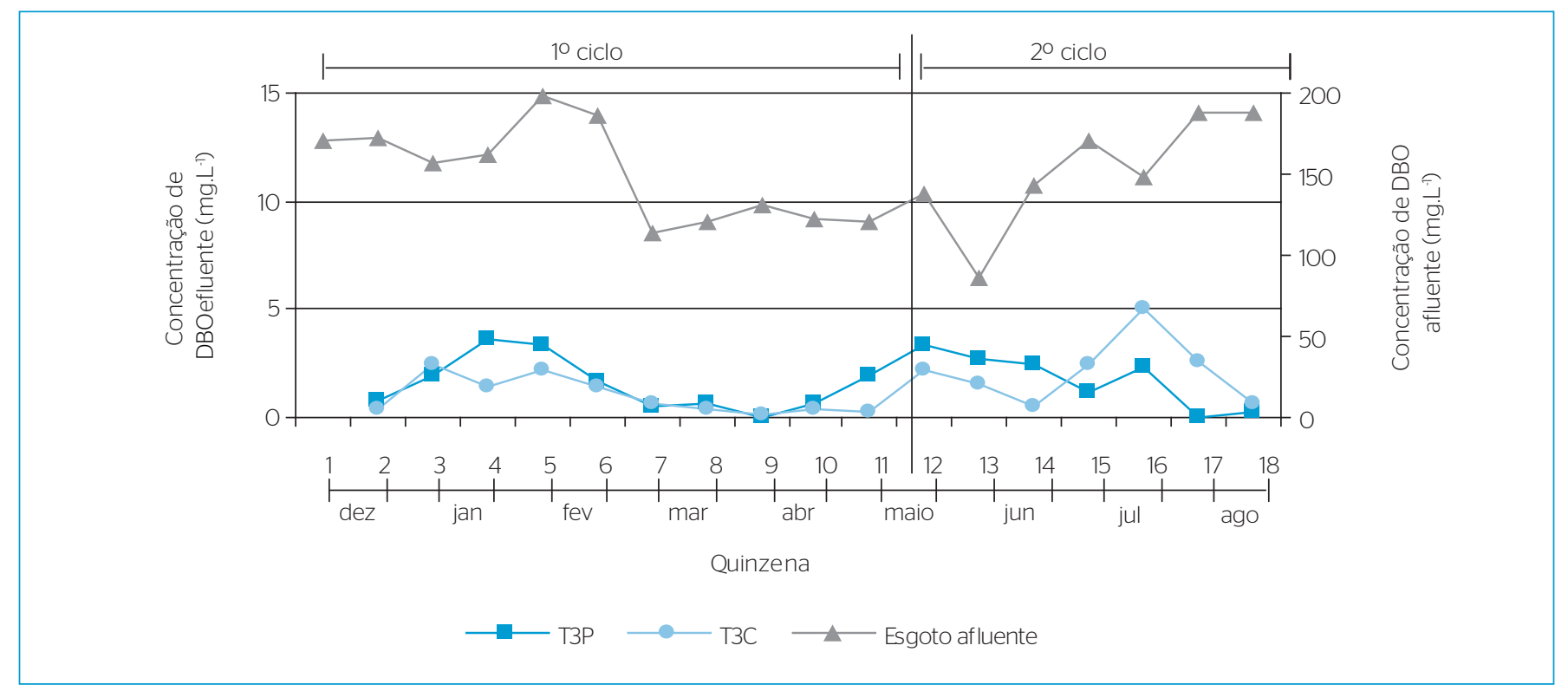

Figura 3 - Concentrações médias de DBO nos afluentes (esgotos) e efluentes das unidades wetlands construídos (T3P) e controles (T3C) do Sistema 3 no período de dezembro de 2005 a agosto de 2006 . 
radicular (MOREIRA \& SIQUEIRA, 2002). Na camada de 5-20 cm, após o segundo ciclo do arroz, não houve diferença significativa entre o wetland construído e o controle, nem entre as taxas de esgoto aplicadas, indicando que nem a presença da planta tampouco o aumento da taxa de aplicação do esgoto contribuiu para um aumento significativo de MO nessa camada (Tabela 4).

Nota-se que houve um maior acúmulo de $\mathrm{MO}$ na camada de $0-5 \mathrm{~cm}$ dos solos dos wetlands construídos e controles, mas não foi muito superior àquele da camada de $5-20 \mathrm{~cm}$.

As alterações nos teores de MO do solo, consequentemente, refletiram no carbono da biomassa microbiana e no carbono perdido pelo solo, quantificado pela respiração basal microbiana (Tabelas 5 a 7).

No final do primeiro ciclo da cultura, a imobilização do $\mathrm{C}$ da biomassa microbiana na camada de $0-5 \mathrm{~cm}$ do solo das unidades controles (sem planta) aumentou significativamente com o aumento da taxa de aplicação. Nos wetlands construídos, a imobilização do $\mathrm{C}$ da biomassa é significativamente maior quando a taxa de aplicação do esgoto passa de 4 para 8 ou para $15 \mathrm{~cm} \cdot \mathrm{d}^{-1}$. Portanto, quando há uma aumento da taxa de 8 para $15 \mathrm{~cm} \cdot \mathrm{d}^{-1}$, a imobilização é insignificante, pois, mesmo aumentando significativamente a atividade microbiana com o aumento da taxa de aplicação, a imobilização microbiana do C

Tabela 3 - Quantidade de Matéria Orgânica adicionada via aplicação do esgoto e aquela remanescente na camada de $0-5 \mathrm{~cm}$ dos solos após o segundo ciclo da cultura do arroz (dezembro de 2005 a setembro de 2006).

\begin{tabular}{|c|c|c|c|c|c|}
\hline \multirow[t]{2}{*}{ Sistema } & \multirow{2}{*}{$\underset{\left(\mathrm{cm}^{\left.-d^{1}\right)}\right.}{\mathrm{q}_{\mathrm{h}}}$} & \multicolumn{2}{|c|}{$\begin{array}{c}\text { Teor de MO } \\
\text { (g MO.kg de solo-1) }\end{array}$} & \multicolumn{2}{|c|}{$\begin{array}{c}\text { MO remanescente no } \\
\text { solo } \\
\text { (g MO.kg de solo-1) }\end{array}$} \\
\hline & & Efluente & $\begin{array}{l}\text { Solo + } \\
\text { esgoto }\end{array}$ & $\begin{array}{l}\text { Wetland } \\
\text { construído }\end{array}$ & Controle \\
\hline 1 & 4 & 0,67 & 8,07 & 14,03 & 11,30 \\
\hline 2 & 8 & 1,41 & 8,81 & 14,83 & 11,43 \\
\hline 3 & 15 & 2,69 & 10,09 & 17,67 & 13,20 \\
\hline Média & & & & $15,51 b^{*}$ & $11,97 a$ \\
\hline
\end{tabular}

MO no solo antes da aplicação do esgoto (g.kg de solo-1) $=7,40$

$\mathrm{q}_{\mathrm{h}}$ : Taxa de aplicação hidráulica do esgoto; MO: Matéria Orgânica.

*Médias seguidas pelas mesmas letras minúsculas nas linhas (comparação entre wetlands e controles) não diferem entre si pelo teste de Tukey $(p<0,05)$

Tabela 4 - Quantidade de Matéria Orgânica do Solo adicionada via aplicação do esgoto e aquela remanescente na camada de $5-20 \mathrm{~cm}$ do solo após o segundo ciclo da cultura do arroz (dezembro de 2005 a setembro de 2006).

\begin{tabular}{|c|c|c|c|c|c|}
\hline \multirow[t]{2}{*}{ Sistema } & \multirow{2}{*}{$\underset{\left(\mathrm{cm} \cdot \mathrm{d}^{-1}\right)}{\mathrm{q}_{\mathrm{h}}}$} & \multicolumn{2}{|c|}{$\begin{array}{c}\text { Teor de MO } \\
\text { (g MO.kg de solo-1) }\end{array}$} & \multicolumn{2}{|c|}{$\begin{array}{c}\text { MO remanescente no } \\
\text { solo } \\
\text { (g MO.kg de solo }{ }^{-1} \text { ) }\end{array}$} \\
\hline & & Efluente & $\begin{array}{c}\text { Solo }+ \\
\text { esgoto }\end{array}$ & $\begin{array}{l}\text { Wetland } \\
\text { construído }\end{array}$ & Controle \\
\hline 1 & 4 & 0,67 & 8,07 & 10,93 & 11,70 \\
\hline 2 & 8 & 1,41 & 8,81 & 12,67 & 10,05 \\
\hline 3 & 15 & 2,69 & 10,09 & 12,10 & 9,33 \\
\hline
\end{tabular}

MO no solo antes da aplicação do esgoto ( g.kg de solo"1) = 7,40

$\mathrm{q}_{\mathrm{h}}$ : Taxa de aplicação hidráulica do esgoto; MO: Matéria Orgânica; MOS: Matéria Orgânica do Solo. entre os sistemas foi aproximadamente semelhante. Isso indica que uma taxa de $15 \mathrm{~cm} \cdot \mathrm{d}^{-1}$ aumentará significativamente a atividade dos microrganismos e imobilizará praticamente a mesma quantidade de carbono na biomassa que seria imobilizada com uma taxa de $8 \mathrm{~cm} \cdot \mathrm{d}^{-1}$.

Nos controles, ao aumentar a taxa de aplicação do esgoto, há um aumento significativo na imobilização do C na biomassa microbiana (Tabela 5). Isso também ocorre com a respiração basal do solo, ou seja, do C perdido do solo (Tabelas 5 e 6) pela atividade dos microrganismos.

No final do segundo ciclo, houve diferença significativa entre os wetlands construídos e controles em imobilizar o C, nas camadas de 0-5 (Tabela 6) e 5-20 cm (Tabela 7), somente no sistema 1. Isso indica que, quando aplicadas taxas pequenas de esgoto, há uma tendência de os solos sem plantas imobilizarem quantidades significativamente maiores do que os solos plantados.

Nos sistemas controle (sem planta), houve menores perdas de carbono pela respiração nas duas camadas $0-5$ e 5-20 cm dos solos, comparados aos sistemas com plantas (wetlands construídos). Esse fato pode ser explicado porque, além de os nutrientes do esgoto serem disponibilizados para os microrganismos, há aqueles dos exsudatos radiculares e os da renovação das raízes do arroz. Deve-se considerar, ainda, que nos wetlands construídos, desde o primeiro ciclo da cultura do arroz (Tabela 2), foram obtidos maiores valores de MO do solo, o que pode ter estimulado a atividade microbiana (Tabelas 5 e 6).

Na camada de 5-20 cm dos solos, os wetlands construídos foram capazes de imobilizar uma quantidade de $\mathrm{C}$ significativamente maior que os controles no sistema 1, que recebeu menor carga de aplicação de esgoto, não diferindo significativamente para os sistemas 2 e 3 .

$\mathrm{O}$ aumento da carga de esgoto aplicada proporcionou maior imobilização do $C$ e maior atividade microbiana na camada de $0-5 \mathrm{~cm}$ dos solos, estimada a partir da liberação de $\mathrm{CO}_{2}$, possivelmente pela maior oferta de C, N e energia mediante a contínua aplicação do esgoto e pela influência da rizosfera.

De acordo com os resultados das Tabelas 5 a 7, dobrando-se a taxa de aplicação de esgoto de 4 para $8 \mathrm{~cm} \cdot \mathrm{d}^{-1}$ nos wetlands construídos (com planta), a atividade microbiana na camada de $0-5 \mathrm{~cm}$ dos solos quase duplicou, aumentando em, aproximadamente, $85 \%$. O acréscimo na atividade microbiana, quando praticamente se dobrou a taxa de 8 para $15 \mathrm{~cm} \cdot \mathrm{d}^{-1}$, foi somente, aproximadamente, de $35 \%$. Isso mostra a tendência de o aumento da taxa de esgoto aplicada passar a não causar efeito significativo ou até mesmo inibir a atividade microbiana em solos plantados.

Nos solos dos controles (sem planta), o aumento da taxa de aplicação de 8 para $15 \mathrm{~cm} \cdot \mathrm{d}^{-1}$ proporcionou um acréscimo na atividade microbiana de $65 \%$. Esses resultados sugerem que um aumento exagerado na taxa de aplicação de esgoto em solos plantados pode inibir a atividade microbiana, enquanto em solos sem planta se tem maior flexibilidade para acréscimos das cargas a serem aplicadas. 
Após o primeiro ciclo da cultura do arroz, na camada de $0-5 \mathrm{~cm}$, e após o segundo ciclo, nas duas camadas de solo (0-5 e 5-20 cm), houve tendência de se obter maior taxa de respiração nos wetlands construídos, pois se tem observado que as raízes estimulam as bactérias no solo pela liberação de oxigênio e pelos exsudatos radiculares em sistemas wetlands (MUNCH; KUSCHK; ROSKE, 2005). Portanto, a maior atividade microbiana em solos plantados ou sem planta ocorre na camada de $0-5 \mathrm{~cm}$ do solo. Isso sugere que os sistemas wetlands construídos de fluxo vertical podem ter melhor desempenho no tratamento que os sistemas wetlands construídos de fluxo subsuperficial de fluxo horizontal.

$\mathrm{Na}$ camada de 5-20 cm, a planta não influenciou significativamente a atividade microbiana, porém, ao aumentar a taxa de aplicação, essa atividade diminuiu significativamente nos solos dos wetlands construídos. Nos solos dos controles, embora a atividade tenha decrescido com o aumento da taxa de aplicação, não houve diferença significativa entre as atividades microbianas dos sistemas 1 e 2, e estas diferiram significativamente daquelas do sistema 3.
Notou-se que o comportamento da atividade microbiana quando aplicado esgoto doméstico para tratamento e cultivos é totalmente diferente daquele no qual se utiliza nitrogênio mineral sob a forma de fertilizantes químicos. Coser (2006), aplicando diferentes doses de nitrogênio mineral na camada de $0-5 \mathrm{~cm}$ do solo, observou que em doses elevadas de $\mathrm{N}$ $\left(120 \mathrm{~kg} \mathrm{ha}^{-1}\right)$ há inibição da atividade microbiana. Convertendo os resultados obtidos, utilizando uma área de $0,26 \mathrm{~m}^{2}$ para hectare, nota-se que foram aplicadas doses de $\mathrm{N}$ bastante superiores nos solos durante o primeiro ciclo da cultura (sistema $1=1.038 \mathrm{~kg} \cdot \mathrm{ha}^{-1}$; sistema $2=3.077 \mathrm{~kg} \cdot \mathrm{ha}^{-1}$; sistema $3=4.154 \mathrm{~kg} \cdot \mathrm{ha}^{-1}$ ) e a atividade microbiana não foi inibida.

Com a continuidade da aplicação do esgoto para tratamento, observou-se que no final do segundo ciclo da cultura do arroz, na camada de $0-5 \mathrm{~cm}$ dos solos dos wetlands construídos, a atividade microbiana aumentou e houve maior liberação dos produtos da mineralização. Isso refletiu no acréscimo da quantidade de $\mathrm{MO}$ e do Carbono Orgânico Total (COT) nos wetlands construídos (Tabela 8), acumulados na

Tabela 5 - Carbono da biomassa microbiana e respiração basal nos wetlands construídos e controles, na camada de 0-5 cm dos solos, após o primeiro ciclo da cultura (dezembro de 2005 a abril de 2006).

\begin{tabular}{|c|c|c|c|c|c|c|c|c|c|}
\hline \multirow{3}{*}{ Sistema } & \multirow{3}{*}{$\begin{array}{l}\text { qh } \\
\left(\mathrm{cm}^{\left.-d^{1}\right)}\right)\end{array}$} & \multicolumn{4}{|c|}{$\begin{array}{c}\text { C Biomassa } \\
(\mathrm{mg} \cdot \mathrm{kg} \text { de solo-1) } \\
\end{array}$} & \multicolumn{4}{|c|}{$\begin{array}{c}\text { C perdido } \\
\left(\mathrm{mg} \mathrm{CO}_{2} \cdot \mathrm{kg} \text { de solo.dia') }\right.\end{array}$} \\
\hline & & \multicolumn{2}{|c|}{ Wetland construído } & \multicolumn{2}{|c|}{ Controle } & \multicolumn{2}{|c|}{ Wetland construído } & \multicolumn{2}{|c|}{ Controle } \\
\hline & & ${\mathrm{mg} \cdot \mathrm{kg}^{-1}}^{-1}$ & $\%$ & $\overline{m g \cdot k^{-1}}$ & $\%$ & mg.kg-1 & $\%$ & $\mathrm{mg} \cdot \mathrm{kg}^{1}$ & $\%$ \\
\hline 1 & 4 & $38,68 \mathrm{aA}$ & 1,13 & 58,71 aA & 1,43 & $6,03 \mathrm{bA} A^{*}$ & 0,18 & 3,34aA & 0,08 \\
\hline 2 & 8 & $82,18 \mathrm{aB}$ & 2,44 & $146,09 \mathrm{bB}$ & 3,77 & $11,17 \mathrm{bB}$ & 0,33 & $8,90 a \mathrm{~B}$ & 0,23 \\
\hline 3 & 15 & $83,77 \mathrm{aB}$ & 1,99 & 109,16 bc & 2,66 & $15,06 \mathrm{aC}$ & 0,36 & $14,66 \mathrm{aC}$ & 0,38 \\
\hline
\end{tabular}

COT ( $\left(\mathrm{kg}^{-1}\right)$ no solo antes da aplicação do esgoto $=4,30$

COT: Carbono Orgânico Total; $\mathrm{q}_{\mathrm{h}}$ : Taxa de aplicação hidráulica do esgoto.

*Médias seguidas pelas mesmas letras minúsculas nas linhas (comparação entre wetlands e controles) e as maiúsculas nas colunas (comparação entre as taxas de aplicação do efluente) não diferem entre si pelo teste de Tukey $(p<0,05)$.

Tabela 6 - Carbono da biomassa microbiana e respiração basal nos wetlands construídos e controles na camada de 0-5 cm dos solos após o segundo ciclo da cultura (dezembro de 2005 a setembro de 2006).

\begin{tabular}{|c|c|c|c|c|c|c|c|c|c|}
\hline \multirow{3}{*}{ Sistema } & \multirow{3}{*}{$\begin{array}{c}\text { qh } \\
\left(\mathrm{cm} \cdot d^{-1}\right)\end{array}$} & \multicolumn{4}{|c|}{$\begin{array}{c}\text { C Biomassa } \\
\left(\mathrm{mg} \cdot \mathrm{kg} \text { de solo }{ }^{-1}\right)\end{array}$} & \multicolumn{4}{|c|}{$\begin{array}{c}\text { C perdido } \\
\left(\mathrm{mg} \mathrm{CO}_{2} \cdot \mathrm{kg} \text { de solo.dia') }\right.\end{array}$} \\
\hline & & \multicolumn{2}{|c|}{ Wetland construído } & \multicolumn{2}{|c|}{ Controle } & \multicolumn{2}{|c|}{ Wetland construído } & \multicolumn{2}{|c|}{ Controle } \\
\hline & & $\mathrm{mg} \mathrm{kg}^{-1}$ & $\%$ & mg.kg-1 & $\%$ & mg.kg ${ }^{-1}$ & $\%$ & $\mathrm{mg} \cdot \mathrm{kg}^{-1}$ & $\%$ \\
\hline 1 & 4 & $22,59 \mathrm{aA}$ & 0,28 & $53,89 \mathrm{bA}$ & 0,82 & 18,33aA & 0,22 & $16,70 \mathrm{aB}$ & 0,25 \\
\hline 2 & 8 & $86,40 \mathrm{aB}$ & 1,00 & $103,05 \mathrm{aB}$ & 1,54 & $25,42 \mathrm{bB}$ & 0,29 & $11,75 a \mathrm{AB}$ & 0,18 \\
\hline 3 & 15 & $126,18 \mathrm{aC}$ & 1,03 & $117,58 \mathrm{aB}$ & 1,53 & $27,64 \mathrm{bB}$ & 0,27 & $12,86 \mathrm{aA}$ & 0,17 \\
\hline
\end{tabular}

COT (g.kg-1) no solo antes da aplicação do esgoto = 4,30

COT: Carbono Orgânico Total.

Tabela 7 - Carbono da biomassa microbiana e respiração basal nos wetlands construídos e controles na camada de 5-20 cm dos solos após o segundo ciclo da cultura (dezembro de 2005 a setembro de 2006).

\begin{tabular}{|c|c|c|c|c|c|c|c|c|c|c|c|}
\hline \multirow{3}{*}{ Sistema } & \multirow{3}{*}{$\mathrm{q}_{\mathrm{h}}\left(\mathrm{cm} \cdot \mathrm{d}^{-1}\right)$} & \multicolumn{2}{|c|}{$\begin{array}{l}\text { COT remanescente no } \\
\text { solo (g.kg de solo-1) }\end{array}$} & \multicolumn{4}{|c|}{$\begin{array}{c}\text { C Biomassa } \\
\text { (mg.kg de solo-1) } \\
\end{array}$} & \multicolumn{4}{|c|}{$\begin{array}{c}\text { C perdido } \\
\left(\mathrm{mg} \mathrm{CO}_{2} \cdot \mathrm{kg} \text { de solo.dia }{ }^{-1}\right) \\
\end{array}$} \\
\hline & & \multirow{2}{*}{$\begin{array}{l}\text { Wetland } \\
\text { construido }\end{array}$} & \multirow{2}{*}{ Controle } & \multicolumn{2}{|c|}{ Wetland construído } & \multicolumn{2}{|c|}{ Controle } & \multicolumn{2}{|c|}{ Wetland construído } & \multicolumn{2}{|c|}{ Controle } \\
\hline & & & & ${\mathrm{mg} \cdot \mathrm{kg}^{-1}}^{-1}$ & $\%$ & mg.kg-1 & $\%$ & mg.kg-1 & $\%$ & mg.kg ${ }^{-1}$ & $\%$ \\
\hline 1 & 4 & 6,37 & 6,80 & $102,73{b A^{*}}^{*}$ & 1,61 & 62,10 aA & 0,91 & $16,00 \mathrm{aC}$ & 0,28 & $12,75 a B$ & 0,19 \\
\hline 2 & 8 & 7,35 & 5,85 & $40,88 \mathrm{aA}$ & 0,56 & $36,05 \mathrm{aA}$ & 0,62 & $10,41 \mathrm{aB}$ & 0,14 & $10,26 a B$ & 0,18 \\
\hline 3 & 15 & 7,03 & 5,43 & $67,20 \mathrm{aB}$ & 0,96 & 45,06 aA & 0,83 & $3,75 \mathrm{aA}$ & 0,05 & 2,47aA & 0,04 \\
\hline
\end{tabular}

COT ( $\left(\mathrm{kg}^{-1}\right)$ no solo Antes da aplicação do esgoto = 4,30

COT: Carbono Orgânico Total; $\mathrm{q}_{\mathrm{h}}$ : Taxa de aplicação hidráulica do esgoto.

*Médias seguidas pelas mesmas letras minúsculas nas linhas (comparação entre wetlands e controles) e as maiúsculas nas colunas (comparação entre as taxas de aplicação do efluente), não diferem entre si pelo teste de Tukey $(p<0,05)$. 
camada de $0-5 \mathrm{~cm}$ dos solos, que pode ter interferido na redução dos percentuais imobilizados na biomassa microbiana e do C liberado na forma de $\mathrm{CO}_{2}$.

As análises estatísticas mostraram que durante o primeiro ciclo da cultura do arroz, na camada de $0-5 \mathrm{~cm}$ dos solos plantados (wetlands construídos) e sem plantas (controles), aplicando-se uma taxa de 8 ou de $15 \mathrm{~cm} \cdot \mathrm{d}^{-1}$, o acúmulo de COT nos solos será semelhante e significativamente maior do que no sistema que recebe menor taxa de aplicação $\left(4 \mathrm{~cm} \cdot \mathrm{d}^{-1}\right)$. Não houve diferença significativa entre os solos dos wetlands construídos e dos controles em acumular COT nos solos. Durante o segundo ciclo, nota-se que não houve influência da taxa de aplicação em acumular COT nos solos, mas os solos dos wetlands construídos acumularam quantidades significativamente maiores do que os solos dos controles (Tabela 8).

O aumento e a redução nos teores de C e MO nos solos também já foram constatados por outros autores. Taha e Malik (2000) observaram, na camada de $0-30 \mathrm{~cm}$ de um solo irrigado com esgoto tratado durante 10 anos, acréscimo insignificante de $\mathrm{C}$ orgânico. Em 20 anos, nesse mesmo solo, houve decréscimo dos teores de C orgânico, refletindo nos teores de MO do solo, reduzindo-os. Rodrigues et al. (2006) observaram redução nos teores de MO no solo depois do cultivo com a aplicação do lodo de esgoto. Foram observados também incrementos nos teores de COT nos solos pela disposição de esgoto doméstico tratado (FRIEDEL et al., 2000).

\section{Quociente metabólico}

De acordo com os resultados da Tabela 9, nas camadas de $0-5 \mathrm{~cm}$ dos solos das unidades controles houve menores perdas de $\mathrm{CO}_{2}$ para a atmosfera e, portanto, maior eficiência metabólica microbiana. Isso pode ser observado pelos menores valores dos quocientes metabólicos e maiores relações C/N (Tabela 10). Segundo Aquino et al. (2005), uma biomassa microbiana eficiente libera menos carbono

Tabela 8 - Carbono Orgânico Total remanescente no solo nos wetlands construídos e controles, na camada de 0-5 cm, após o primeiro e o segundo ciclos do arroz.

\begin{tabular}{l|c|c|c|c|c|c|c}
\multirow{2}{*}{ Sistema } & \multirow{2}{*}{$\begin{array}{c}\mathrm{q}_{\mathrm{h}} \\
\left(\mathrm{cm} \cdot \mathbf{d}^{-1}\right)\end{array}$} & $\begin{array}{c}\text { Wetland } \\
\text { construido }\end{array}$ & Controle & Média & $\begin{array}{c}\text { Wetland } \\
\text { construído }\end{array}$ & $2^{\circ}$ ciclo \\
\cline { 3 - 8 } & 4 & 3,43 & 2,80 & $3,12^{\mathrm{a}}$ & 8,17 & 6,57 & $7,37 \mathrm{~A}$ \\
\hline 2 & 8 & 3,37 & 3,87 & $3,62 \mathrm{AB}$ & 8,63 & 6,67 & $7,65 \mathrm{~A}$ \\
\hline 3 & 15 & 4,20 & 4,10 & $4,15 \mathrm{~B}$ & 10,27 & 7,67 & $8,96 \mathrm{~A}$ \\
\hline Média & & $3,58 \mathrm{a}$ & $3,66 \mathrm{a}$ & & $9,02 \mathrm{~b}$ & $6,97 \mathrm{a}$ & \\
\hline
\end{tabular}

COT (g.kg') no solo antes da aplicação do esgoto - 4,3

COT: Carbono Orgânico Total; $\mathrm{q}_{\mathrm{h}}$ : Taxa de aplicação hidráulica do esgoto.

* Médias seguidas pelas mesmas letras minúsculas nas linhas (comparação entre wetlands e controles) e as maiúsculas nas colunas (comparação entre as taxas de aplicação do efluente) não diferem entre si pelo teste de Tukey $(p<0,05)$

Tabela 9 - Quociente metabólico $\left(\mathrm{qCO}_{2}\right.$ ) nas camadas de 0-5 e 5-20 cm dos solos dos wetlands construídos e controles dos sistemas 1, 2 e 3 após o segundo ciclo da cultura do arroz.

\begin{tabular}{|c|c|c|c|c|c|c|c|}
\hline \multirow{4}{*}{ Sistema } & \multirow{4}{*}{$\underset{\left(\mathrm{cm}_{\mathrm{h}} \cdot \mathrm{d}^{-1}\right)}{\mathrm{q}}$} & \multicolumn{6}{|c|}{$\mathrm{qCO}_{2} \mathrm{mg} \mathrm{C} / \mathrm{kg}$ solo } \\
\hline & & \multirow{2}{*}{\multicolumn{2}{|c|}{$\begin{array}{c}10 \text { ciclo (dez/05-abr/06) } \\
\text { Profundidade: } 0-5 \mathrm{~cm}\end{array}$}} & \multicolumn{4}{|c|}{$2^{\circ}$ ciclo (maio/06-set/06) } \\
\hline & & & & \multicolumn{2}{|c|}{ Profundidade: $0-5 \mathrm{~cm}$} & \multicolumn{2}{|c|}{ Profundidade: $5-20 \mathrm{~cm}$} \\
\hline & & $\begin{array}{c}\text { Wetland } \\
\text { construido }\end{array}$ & Controle & $\begin{array}{l}\text { Wetland } \\
\text { construído }\end{array}$ & Controle & $\begin{array}{l}\text { Wetland } \\
\text { construído }\end{array}$ & Controle \\
\hline 1 & 4 & 0,16 & 0,06 & 0,81 & 0,31 & 0,16 & 0,21 \\
\hline 2 & 8 & 0,14 & 0,06 & 0,29 & 0,11 & 0,25 & 0,28 \\
\hline 3 & 15 & 0,18 & 0,13 & 0,26 & 0,11 & 0,06 & 0,05 \\
\hline
\end{tabular}

Tabela 10 - Relação C/N da biomassa microbiana nos solos das camadas de 0-5 e 5-20 cm das unidades wetlands construídos e controles dos sistemas 1, 2 e 3 após o segundo ciclo da cultura do arroz.

\begin{tabular}{|c|c|c|c|c|c|c|c|}
\hline \multirow{4}{*}{ Sistema } & \multirow{4}{*}{$\underset{\left(\mathrm{cm}_{\mathrm{h}} \mathrm{d}^{-1}\right)}{\mathrm{q}_{\mathrm{f}}}$} & \multicolumn{6}{|c|}{ Relação C/N da biomassa } \\
\hline & & \multirow{2}{*}{\multicolumn{2}{|c|}{$\begin{array}{l}10 \text { ciclo (dez/05-abr/06) } \\
\text { Profundidade: } 0-5 \mathrm{~cm}\end{array}$}} & \multicolumn{4}{|c|}{$2^{\circ}$ ciclo (maio/06-set/06) } \\
\hline & & & & \multicolumn{2}{|c|}{ Profundidade: $0-5 \mathrm{~cm}$} & \multicolumn{2}{|c|}{ Profundidade: $5-20 \mathrm{~cm}$} \\
\hline & & $\begin{array}{l}\text { Wetlands } \\
\text { construídos }\end{array}$ & Controles & $\begin{array}{l}\text { Wetlands } \\
\text { construídos }\end{array}$ & Controles & $\begin{array}{l}\text { Wetlands } \\
\text { construídos }\end{array}$ & Controles \\
\hline 1 & 4 & 4,56 & 8,94 & 2,56 & 4,93 & 10,95 & 8,03 \\
\hline 2 & 8 & 8,59 & $26,84^{*}$ & 5,02 & 5,61 & 2,85 & 7,27 \\
\hline 3 & 15 & 6,26 & 9,28 & 3,79 & 5,27 & 5,02 & 4,40 \\
\hline
\end{tabular}

*Dado duvidoso, pois está muito diferente dos outros valores. Pode ter ocorrido erro de medida ou de anotação. 
na forma de $\mathrm{CO}_{2}$ pela respiração, mas incorpora carbono em sua constituição, aumentando a sua massa microbiana, evitando assim a perda de $\mathrm{C}$ do solo.

Em solos de wetlands construídos para tratamento de esgotos domésticos, quanto maior a atividade microbiana, melhor, pois isso garantirá a maior remoção possível de MO presente nos esgotos, mineralizada e removida do esgoto.

Os maiores quocientes metabólicos dos solos dos wetlands construídos indicam maior proporção da biomassa microbiana metabolicamente ativa e em crescimento nos solos (FISK \& FAKEY, 2001) e maior liberação de $\mathrm{CO}_{2}$ para a atmosfera.

Em sistemas naturais projetados para tratamento de esgotos domésticos, é importante que haja menor imobilização de $\mathrm{C}$ na biomassa microbiana, porque uma grande imobilização poderá indicar formação do biofilme (película microbiana) que cresce aderido às partículas do solo e contribui para o processo de colmatação. Os sistemas wetlands construídos, portanto, apresentam uma vantagem em relação aos controles, uma vez que a presença da planta tem importância no controle da colmatação do solo.

A maior liberação de $\mathrm{CO}_{2}$ para a atmosfera nos solos dos sistemas wetlands construídos poderá ser compensada a partir da fixação do $\mathrm{CO}_{2}$ atmosférico pela planta via fotossíntese, que estabelecerá o equilíbrio do sistema. Assim, não haverá liberação excessiva de $\mathrm{CO}_{2}$ para a atmosfera, fato que contribuiria para aumentar os problemas atmosféricos como, por exemplo, o efeito estufa.

\section{CONCLUSÕES}

Durante o período de operação dos sistemas, foi observada lixiviação de concentrações mínimas de DBO para os efluentes tratados, que apresentaram características de rios de Classes 1 e 2. O processo de remoção da MO dos esgotos predominante foi o de mineralização pelos microrganismos e acúmulo no solo. Inicialmente, houve uma redução dos teores de MO presente no solo, que já era distrófico, passando a aumentar paulatinamente com a constante aplicação dos esgotos domésticos para tratamento, promovendo o início da fertilização do solo. Nas unidades wetlands construídos, as raízes contribuíram para uma maior atividade microbiana e uma menor imobilização microbiana de $\mathrm{C}$, o que pode evitar a rápida colmatação do solo, pois nos sistemas plantados (wetlands construídos) poderá haver uma redução do biofilme (película microbiana) formado pela sua rápida mineralização pelos microrganismos. Foi observado também que:
- apesar de ter ocorrido inicialmente um decréscimo nos teores de MO e COT nos solos, ao longo do período de operação dos sistemas wetlands constituídos e controles, houve um gradativo aumento dos teores de MO e COT nos solos, passando a melhorar as suas propriedades. Isso pode proporcionar a fertilização do solo, tornando-o um reservatório de nutrientes, o que possibilitará o seu aproveitamento para plantio de outras culturas, evitando o uso de fertilizantes químicos ou até mesmo usando esse solo na recuperação de áreas degradadas quando ele perder a capacidade de tratamento dos esgotos;

- com o aumento da taxa de aplicação do esgoto, há uma maior atividade microbiana na camada de $0-5 \mathrm{~cm}$ do solo, independentemente de o solo estar plantado ou sem planta. Esse aumento foi significativamente maior após os primeiros cinco meses de aplicação dos esgotos (primeiro ciclo da cultura do arroz) e após nove meses de operação dos sistemas (segundo ciclo da cultura do arroz). A diferença dessa atividade entre os sistemas que receberam maiores taxas de aplicação dos esgotos já não foi significativa, indicando que uma taxa muito grande poderá inibi-la;

- a atividade microbiana nas camadas de $0-5 \mathrm{~cm}$ dos solos plantados (wetlands construídos) foi significativamente maior do que nos solos sem planta (controles). Isso sugere que houve grande atividade dos microrganismos rizosféricos no processo de tratamento do esgoto afluente, possivelmente pelo fato de os wetlands construídos terem maior quantidade de amônio devido à contribuição do $\mathrm{N}$ dos compostos liberados pelas substâncias exsudadas pelas raízes do arroz. As bactérias nitrificantes, então, têm uma maior atividade, que, somada à dos microrganismos rizosféricos, favorece o aumento da respiração microbiana nos sistemas wetlands construídos. Essa maior atividade contribuiu para uma menor imobilização microbiana em função da rápida ciclagem de nutrientes;

- a aplicação de taxas elevadas de esgoto em áreas pequenas pode reduzir a eficiência do tratamento dos esgotos em curto prazo, uma vez que limita a atividade microbiana, cujo papel é fundamental nos processo de remoção de poluentes;

- a compreensão da matriz Solo-Planta-Microrganismos é muito complexa e requer estudos sistemáticos e constantes por um período prolongado para que se possa compreender melhor os mecanismos bioquímicos que ocorrem durante o tratamento nesse tipo de sistema. Assim, poder-se-á estabelecer uma configuração ótima que garanta a eficiência dos sistemas wetlands durante o período para o qual for projetado. 


\section{REFERÊNCIAS}

ALEF, K. \& NANNIPIERRE, P. (1995). Methods in applied soil microbiology and biochemistry. London: Academic Press.

APHA-AWWA-WPCF. (1985) Standard Methods for the Examination of Water and Wastewater. 16th edition, Washington, DC, American Public Health Association, 1268 p.

AQUINO, A.M.; SILVA, E.M.R.; SAGGIN JUNIOR, O.; RUMJANEK, N.; De POLLI, H.; REIS, V.M. (2005) A biota do solo e processos relevantes num novo contexto da agricultura. In: Recomendações para ão e manejo da fertilidade do solo no estado do Acre. Rio Branco, v. prelo, cap. 4.

BRASIL. (2005) Resolução CONAMA n० 357, de 17 de março de 2005. Estabelece a classificação de águas doces, salobras e salinas. Diário Oficial da República Federativa do Brasil, 24 p.

BRASIL. (2011) Resolução CONAMA no 430, de 13 de maio de 2011. Condições e padrões de lançamento de efluentes e complementa e altera a Resolução CONAMA no 357/05. Diário Oficial da República Federativa do Brasil, 8 p.

CALIJURI, M.L.; BASTOS, R.K.X.; BEVILACQUA, P.D.; CAPELETE, B.C.; MAGALHÃES, T.B. (2009) Operação de partida de sistemas wetlands construídos como pós-tratamento de efluentes (esgotos sanitários) de reatores UASB. In: $24^{\circ}$ Congresso Brasileiro de Engenharia Sanitária e Ambiental.

COSER, T.R. (2006) Doses de Nitrogênio e seu efeito nos indicadores microbiológicos de qualidade de solo na cultura da cevada. Dissertação (Mestrado) - Faculdade de Agronomia e Medicina Veterinária, Universidade de Brasília, Brasília, DF, 81p.

EPA. (2006) Constructed wetlands treatment of municipal wastewaters. EPA/625/R/O1O, Cincinnati, Ohio, U.S.A, 165p.

FIA, R.; MATOS, A.T.; QUEIROZ, M.E.L.R.; CECON, P R.; FIA, F.R.L. (2010) Desempenho de sistemas alagados no tratamento de águas residuárias do processamento dos frutos do cafeeiro. Revista Brasileira de Engenharia Agrícola e Ambiental, v. 14, n. 12, p. 1323-1329.

FISK, M.C. \& FAKEY, T.J. (2001) Microbial biomass and nitrogen cycling responses to fertilization and litter removal in young northern hardwood forest. Biochemistry, v. 53, n. 201-223.

FRIEDEL, J. K.; LANGER, T.; SIEBE, C; STAHR, K. (2000) Effects of long-term waste water irrigation on soil organic matter, soil microbial biomass and its activities in central Mexico. Biololy and Fertility of Soils, v. 31, n. 5, p. $414-421$.

KUZYAKOV, Y.; STAHR, J.K.; FRIEDEL, K. (2000) Review of mechanisms and quantifcation of priming effects. Soil Biology Biochemistry, v. 32, n. 11-12, p. 1485-1498.

MARTINAZZO, R. (2006) Diagnóstico da Fertilidade de Solos em Áreas sob Plantio Direto Consolidado. Dissertação (Mestrado) - Programa de Pós-Graduação em Ciência do Solo, Centro de Ciências Rurais, Universidade Federal de Santa Maria.
MEIRA, C.M.B. (2004). Utilização de Terras Úmidas no Tratamento de Águas Superficiais Poluidas: Influência do Tipo de Leito e de Macrófita. Tese (Doutorado) - Departamento de Engenharia Civil e Ambiental, Universidade Federal de Campina Grande, Campina Grande, João Pessoa.

MORARI, F. \& GIARDINI, L. (2009) Municipal wastewater treatment with vertical flow constructed wetlands for irrigation reuse. Ecological Engineering, Jornal Elsevier, v. 35, p. 643-653.

MOREIRA, F.M.S. \& SIQUEIRA, J.O. (2002) Microbiologia e bioquímica do solo. Lavras: Editora UFLA, 625 p.

MUNCH, C.; KUSCHK, P.; ROSKE, I. (2005) Root stimulated nitrogen removal: only a local effect or important for water treatment? Water Science and Technology, v. 51, n. 9. p. 185-92

NASCIMENTO, L.V. (1998). Análise dos Padrões de Qualidade das Águas e de Lançamento de efluentes líquidos estabelecidos pela Resolução CONAMA no 20/1986. Uma abordagem para águas doces. Dissertação (Mestrado em Saneamento, Meio Ambiente e Recursos Hídricos) Universidade Federal de Minas Gerais, Belo Horizonte, 123 p.

RODRIGUES, L.S.; BALDINI, V.L.D.; REIS, V.M.; BALDINI, J.I. (2006) Diversidade de bactérias diazotróficas endofíticas dos gêneros Herbaspirillum e Burkholderia na cultura do arroz inundado. Pesquisa Agropecuária Brasileira, v. 41, n. 2.

SILVA, F.A.M. (2005) Qualidade de compostos orgânicos produzidos com resíduos do processamento de plantas medicinais. Tese (Doutorado) Departamento de Energia na Agricultura, Faculdade de Ciências Agronômicas, Universidade Estadual Paulista “Julio de Mesquita Filho”, 102 p.

SKLARZ, M. Y.; GROSS, A.; YAKIREVICH, A.; SOARES, M.I.M. (2009) A recirculating vertical flow constructed wetland for the treatment of domestic wastewater. Desalination. Jornal Elsevier, v. 246, p. 617-624.

TAHA, I. M. \& MALIK, N. (2000) Effect of treated sewage water on soil Characteristics. In: Proc. International Conf. on Wastewater Treatment and Reuse Adapted to Mediterranean Area, p. 25-28.

TRANG, N. T. D.; KONNERUP, D.; SCHIERUP, H.H.; CHIEM, N.H.; TUAN, L.A.T.; BRIX, H. (2O10) Kinetics of pollutant removal from domestic wastewater in a tropical horizontal subsurface flow constructed wetland system: Effects of hydraulic loading rate. Ecological Engineering, Jornal Elsevier, v. 36, p. 527-535

TRUU, M.; JUHANSON, J.; TRUU, J. (2009) Microbial biomass, activity and community composition in constructed wetlands. Science of the total environment, v. 407, p. 3958-3971.

VANCE, E.D.; BROOKES, P.C.; JENKINSON, D.S. (1987) An extraction method for measuring soil microbial biomass C. Soil Biology Biochemistry, v. 19, p. 703-707.

VYMAZAL, J. (1998). Types of constructed wetlands for wastewater treatment. Proceedings: 6th International Conference on Wetlands Systems for Water Pollution Control, p. 150-166. 